\title{
Simulation of Zahavi's Handicap Principle
}

\author{
A. Ticona and T.J.P. Penna \\ Instituto de Física, Universidade Federal Fluminense \\ Av. Litorânea s/n, Boa Viagem, Niterói 24210-340, RJ, Brazil
}

Received on 2 May, 2003

\begin{abstract}
We study the plausibility of the handicap principle, using a bit-string model to represent both the genoma and the phenotype of the individuals of a population. We find that the distribution of genoma of population selforganizes due to the natural selection. The phenotype represents some trait of the interaction of individuals with others and with the environment so, it also suffers the pressure of natural selection. The handicap is introduced in sexual selection. At time of reproduction, females compare males according to the phenotype, choosing the one who has a phenotype representing the greatest handicap. Our results show that in this way females are able to see the quality of their possible mates and males have no possibility to cheat due to pressure of natural selection.
\end{abstract}

\section{Introduction}

The development of exaggerated secondary sexual ornaments for sexual selection was already documented by Darwin $[1,2]$. He tried to explain them by the cumulative effect of females preference for certain male types. He suggested that the disadvantage to male survival induced by these characters are compensated by the preference of females to males bearing them. But Darwin did not provide a hypothesis for the origin or maintenance of these preferences. Fisher [1,2] suggests that there is a correlation between the character preferred by females and the quality of the male. O'Donald $[1,2]$ pointed out that a small increase in the male trait results in a small lost of viability but a large gain in mating advantage in the sexual selection process.

Females tend to be the choosier sex [3], because males are able to produce millions of sperm whereas females' eggs are few and far between. So males tend to compete for females and not vice versa. Sexual selection can be classified in two sorts: male-to-male fight and female choice [4]. In species where males are less aggressive, females choose their mates according to sexual secondary characteristics, such as ornamental plumage or courtship displays [3]. Benefits for females may be direct, such a safe copulation or increased fertility, but it also has been reported the interest of females in indirect genetic benefits, such as better offspring, seems to be more attractive to females [5].

There are many works trying to explain how preference for some traits has been developed. Kirkpatrick $[2,6]$ suggested that these characteristics do not have any correlation with the quality of males, and there is a multiple possible evolutionary equilibria. Then any kind of trait could be chosen as the preferred one by females and not necessarily one which represents a disadvantage to males. It also has been suggested the existence of an allele encoding the preference for some trait in females and another one for the trait in males $[1,6,7,10]$. Then, an increase in the frequency in the preference allele will also result in an increase in the frequency of the preferred trait allele, what is called a runaway process $[1,2]$. This process was used by Fisher in order to explain the development of these secondary sexual ornaments.

Zahavi $[1,8,9]$ proposed the handicap principle, where secondary sexual ornaments act as handicaps for males in order to test their quality. He stated that when the phenotype may affect the reproductive potential, it may be useful to test individuals for their phenotypic fitness and not only for their genotype. Under such circumstances, if handicaps act as a test, they may be useful characters even in a population at its optimal fitness among individuals which are similar to one another in their genetic constitution. So, in agreement with Ficher's statement, the secondary sexual ornaments are developed as signals of males' quality, giving advantages for both sexes, i.e. males show their true quality and females are able to select a good quality male as a function of its handicap. These handicaps do not allow cheating because of their cost in survival, so they work as honest signals $[1,8,9,10,11]$. Of course species that practice parental care can not afford to use this kind of signals, because males with handicaps have their survivorship reduced.

The handicap principle has been used to explain other strange behavior in animal and even in human relationship $[9,10,11,12]$. This principle can also be applied in economics due to interaction between sellers and buyers $[9,10]$. In this work we show how the handicap principle works in sexual selection. We use a bit-string model to represent genoma and phenotype of individuals. Genoma selforganizes due to natural selection. We use the phenotype to represent some trait which influences survival due to the 
interaction of individuals with environment and other individuals of population, so, we use it in sexual selection representing the handicap for males. Then we show results of males' phenotype and the variation of quantity of males in population, as a consequence of pressure of sexual selection. Finally we discuss our results in order to understand the importance of the handicap principle in sexual selection and the implications of honesty in signaling.

\section{The Penna Model and the Handi- cap Principle}

The Penna model is based on an age-structured population dynamics [13]. In the present work we use the sexual version [14], but instead of representing individuals by two bit-string, we use only one. This corresponds to the two bit-string model in the extreme case of considering all the position as dominant [14], although this way, diversity of genoma is decreased. Each individual in the population is represented by a computer word (a string containing 0 's and 1 's) with a given length $A$. Each position represents a time interval in the individuals life. At every time one position of the bit-string is read, if that position is set to 1 , the individual starts to suffer the effects of an inherited disease from that "year" on until its death.

The model is based in the accumulation of bad mutations, so when an individual accumulates a limit number $T$ of deleterious mutations, dies at that age. An individual may also die because of intra-specific competition for the finite resources of the environment or because of the actions of predators. This interaction is represented by the logistic Verhulst factor, defined as: $V=N(t) / N_{\max }$. Where $N(t)$ is the current population size and $N_{\max }$ is the maximum population size the environment can support. At each time step and for each individual a random number between 0 and 1 is generated and compared with $V$, if it is smaller than $V$, the individual dies independently of its age or genome.

Every time step each female with age equal to or greater than the minimum reproductive age $R$ randomly chooses a male with age also equal to or greater than $R$ to mate, generating $H$ offspring. The offspring genome is constructed as follows: the mother and father genomes are cut in a random position, generating four bit string pieces. Two complementary pieces, each one coming from one of the original strings, are joined to form the offspring string. After this, $M_{g}$ random mutations are included. Only deleterious mutations are considered. The sex of the newborn is randomly chosen. All this process of testing the survival of each individual and the process of reproduction, both applied over the whole population, represents a time step, i.e. one year in the simulation.

Rather than random deaths caused by the intra-specific competition, we expect to have also a selective value. The probability of their occurrence should depends on the fitness of the individual to the environment, and this fitness a function of the match between the individual's phenotypic expression of genetically acquired traits and a phenotype ideally adapted to its habitat. So we introduce an extra bitstring with the same length $A$ in order to represent the phenotype of individuals. This case is similar to the original one used by Martins, de Oliveira and de Medeiros, but with all position as dominant [15] (diversity is decreased again). The new portion of the phenotype represents some individual trait, such as the size of the beak or the length of its tail. It is not age-structured, so we read the whole word once for each individual generating a fitness function $f(n)$, where $n$ is the quantity of 1-bits in this string.

The fitness function encapsulates the selective value of a particular phenotype and restricts the multi-dimensional space of the interaction between the individual and its environment to one single dimension. Now the probability of death by intra-specific competition at each time is given by $V / f(n)$. So the interaction with other individuals is no longer uniform, and depends on the particular genetically acquired configuration of each individual. The function is given by $f(n)=1-(A-n) / B$, where $B$ is a boost factor that can be used to control the intensity of the selective pressure. This fitness function express an environment where the ideal phenotype would be composed entirely of 1-bits.

Although in the original model, the rules to generate the phenotype, at time of reproduction, were the same to the genoma, in the present work, this string is copied from the father if the offspring is a male, otherwise from the mother, with $M_{p}$ random mutation. In this case mutations can be bad or good with the same probability. Using this rule to generate the phenotype, we try to represent some traits in species that show differences among sexes, such as tail in peacock or horns in great deers.

Now we impose a new condition in sexual selection. Females select their mates as a function of the quantity of 1bits in the males' phenotype string. Contrary to natural selection, we impose that females prefer males with less number of 1-bits in their phenotype. This way, we introduce the handicap in the model, i.e. a male with few 1-bits in their phenotype has its survival capacity reduced, but at time of reproduction, it has the preference of females. An example of this kind of behavior, is the peacock, where females prefer males with a long tail. In the other hand, a long tail represents for males, a reduction in their survival capacity, because its harder to avoid dangers carrying a long showy tail.

\section{Results}

We begin the simulations generating randomly genoma and phenotype of individuals, then after around 20000 time steps we take averages during the following 10000 time steps. The values used for the simulations are: 


$\begin{array}{ll}\text { Maximum population size } & N_{\max }=35 * 10^{5} \\ \text { The bit string length } & A=16 \\ \text { Limit of deleterious mutations } & T=2 \\ \text { Minimum reproduction age } & R=6 \\ \text { Mutation rate of genoma } & M_{g}=1 \\ \text { Mutation rate of phenotype } & M_{p}=1 \\ \text { Birth rate } & H=1 \\ \text { Boost factor } & B=16\end{array}$

In our simulations we begin with $7 * 10^{4}$ males and the same quantity of females, so we are sure that all the possible combinations $\left(2^{16}\right)$ will be present at the beginning. Natural selection leaves only the best fitted genomas i.e. the ones with less quantity of 1-bits before the reproduction age. When we apply the standard Verhulst factor, the original quantity of phenotypes is almost totally kept and the mean quantity of 1-bits is 8 (Fig. 1). The fitness function $f(n)$ makes that only the phenotypes with more 1-bits to be kept, and the mean quantity of 1-bits is always higher than 8 (Fig. $1)$.

Males are under the pressure of natural and sexual selection, then the distribution of 1-bits in the phenotype has a mean value smaller than 8 , as can be seen in Fig. 1. In this example, females are allowed to chose between 2 males. In this figure, we also plot the values of the standard and the modified Verhulst factor, as a function of 1-bits in the phenotype string.

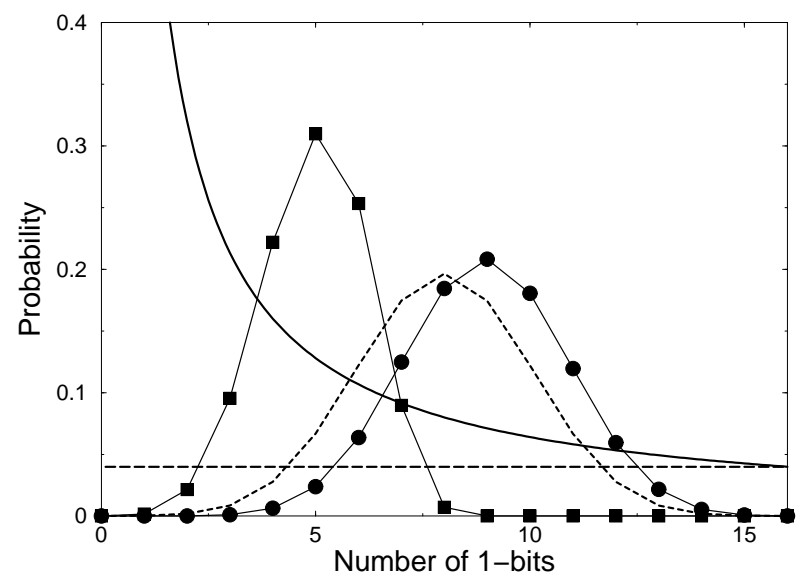

Figure 1. Distribution of 1-bits in phenotype string: females (full circles), males (full squares) and without $f(n)$ (dotted line). Verhulst factor: standard (dashed line) and modified (full line).

As it can be seen, although it is more convenient to have a phenotype with more 1-bits, the modified Verhulst factor allows males to survive with few 1-bits, of course, the cost is greater when the number of 1-bits in the phenotype, gets closer to 0 , where the survival probability is zero. The distribution of the phenotypes changes when sexual pressure is higher, i.e. when females perform selection comparing a lot of males, before choosing the mate. In this case, the greater the number of males compared, the closer to 0 the mean value of 1-bits in the phenotype gets, as we can see in Fig. 2.
In Fig. 2, we can see a change in the slope of the mean value of 1-bits, this is due to the lowest quantity of 1-bits in the phenotype allowed by the modified Verhulst factor. This factor does not allow to have a phenotype completely full of 0-bits, because these kind of individuals would not survive. So it does not matter how strong is the sexual pressure, there will never be a male with perfect phenotype. This change in the slope can also be seen in Fig. 3, where we plotted the quantity of phenotypes in population.

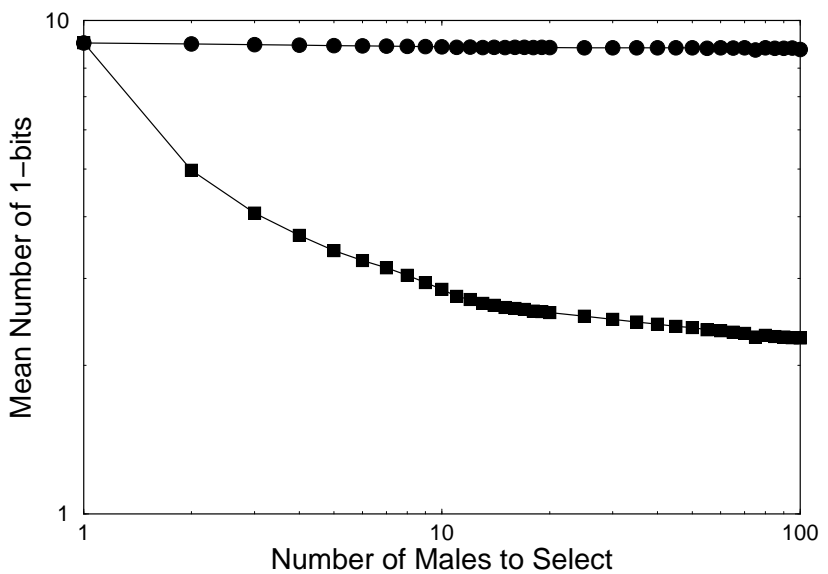

Figure 2. Logarithm of the mean value of 1-bits in phenotype string as a function of the logarithm of the number of compared males by females in reproduction. Males phenotype (full squares) and females phenotype (full circles).

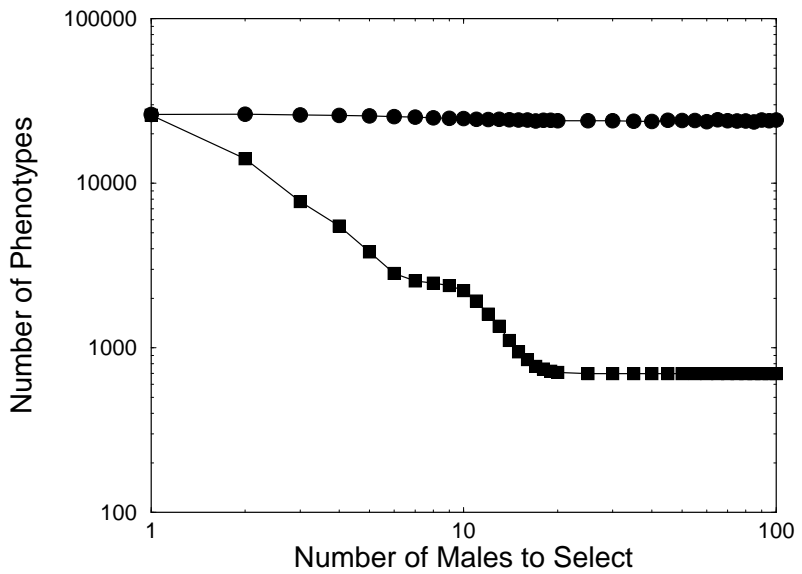

Figure 3. Logarithm of the quantity of phenotypes present in population as a function of the logarithm of the number of compared males by females in reproduction. Males phenotypes (full squares) and females phenotypes (full circles).

It can be seen, that the quantity of phenotypes in population for males, reduces when the pressure of sexual selection increases. This means that the distribution of 1-bits in the phenotypes gets sharp, pushed for one side by natural selection and for the other side by sexual selection so, only males with the phenotype very similar to the "perfect" one, are present in the population. Of course this pressure has consequences in the males' survival, i.e. when the sexual pressure is too high, the males are living too close of the limit allowed by natural selection and the quantity of males 
in population reduces, as can be seen in Fig. 4. We see again a change in the behavior, at a given quantity of males compared.

\section{Discussion}

In our model the modified Verhulst factor represents the quality of individuals therefore, in this work we have considered all the individuals with the same quality. Then we

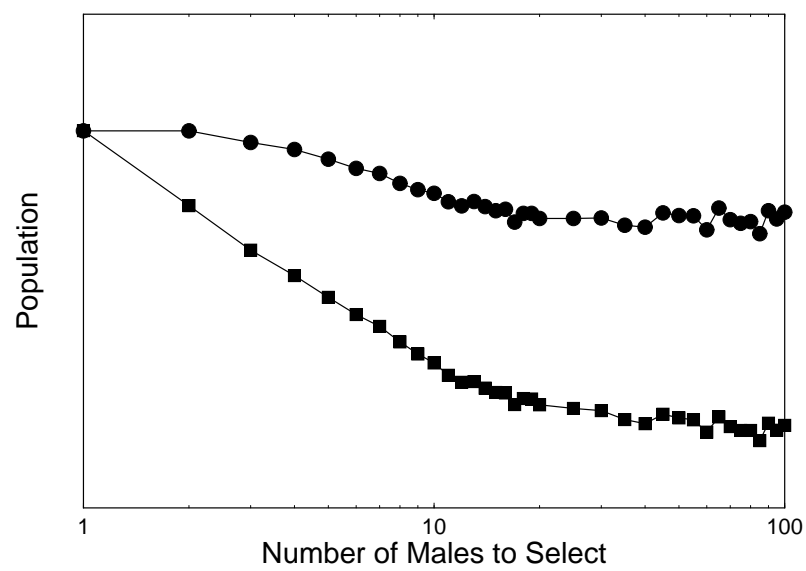

Figure 4. Logarithm of the population as a function of the logarithm of the number of compared males by females in reproduction. Males (full squares) and females (full circles).

can see that the function of the handicap, is to show the quality of an individual, as suggested by Zahavi [1, 8, 9]. If we would have males with different qualities and only natural selection, females could not differentiate them, because, all the phenotypes would have a similar distribution, which did not represent any danger in survival. But when sexual selection is strong, all males are obligated to show its true quality, living as closer as is possible, to the limit allowed by natural selection.

The signals represented in this case by the distribution of 1-bits in the phenotype string, must be honest, because natural selection does not allow to cheat. For example, an individual with the quality used in this work, can not have its phenotype with only 0-bits, although it would have a lot of benefits in reproduction, when its survival probability was tested, it would die due to the Verhulst factor. The change in behavior of the quantities showed in the figures, also justifies honest signaling, because we see that although we increase pressure, the modifications in the mean phenotype only continue changing in order to agree females' preference, until the limit allowed by their quality.

The mutation rate of the phenotype $M_{p}$ influences the standard deviation of the distribution of 1-bits and also the mean value. For example, in the case of only natural selection, if $M_{p}$ is smaller than 1 the mean value tends to 16 and the distribution gets sharper. We used $M_{p}=1$ in order to have a greater diversity of phenotypes as is possible. Other values of this probability would change mostly the quantity of phenotypes presents at equilibrium, leading to sharper distribution. But the results of the mean quantity of 1-bits in the phenotype would not differ with the results showed here, so the principal consequences of handicap and honesty would be kept.

The boost factor $B$ controls the intensity of the selective pressure, this intensity is a decreasing function of $B$. In the present work we use the minimum value allowed $B=A$, so the intensity of pressure is the maximum. For smaller values the quality function represented by the modified Verhulst factor, is meaningless. For greater values the quality allows to have all the phenotypes, even those ones with no 1-bits in the string. In that case we would not be able to see the necessity of honesty of signaling.

As we said when we explained the model of the phenotype, we are reducing the multi-dimensional space of the interaction between the individual and its environment to one single dimension. This way we are not considering all the possibles female preferences that can also have a stable solution. Anyway, our model justifies the kind of preferences that produce a handicap, because of the benefits that represents in sexual selection, that it is why we thing, this kind of signaling is more frequently used when there is a competing interaction between individuals.

\section{Acknowledgments}

We thank P.M.C. de Oliveira, C. Tsallis and D. Stauffer for helpful discussions. This work was partially supported by CLAF and Brazilian agencies CAPES, CNPq and FAPERJ.

\section{References}

[1] A. Zahavi, J. Theor. Biol. 53, 205 (1975).

[2] M. Kirkpatrick, Evolution 36, 1 (1982).

[3] L. A. Dugatkin, J.G. Godin, Scientific American 278, 56 (1998).

[4] S. G. F. Martins, T. J. P. Penna, Inter. Journal of Modern Physics C 9, 491 (1998).

[5] M. Petrie, Letters to Nature 371, 598 (1994).

[6] M. Kirkpatrick, The American Naturalist 127, 222 (1986).

[7] H. K. Reeve, D. W. Pfennig, Proc. Natl. Acad. Sci. 100, 1089 (2003).

[8] A. Zahavi J. Theor. Biol. 67, 603 (1977).

[9] A. Zahavi, A. Zahavi, The Handicap Principle, (Oxford University Press, 1997).

[10] A. Grafen, J. Theor. Biol. 144, 517 (1990).

[11] A. Lotem, Etología 3, 209 (1993).

[12] G. Roberts, Proc. R. Soc. Lond. B 265, 427 (1998).

[13] T.J.P. Penna, J. Stat. Phys. 78, 1629 (1995).

[14] A.T. Bernardes, Physica A 230, 156 (1996); D. Stauffer, P.M.C. de Oliveira, S. Moss de Oliveira, and R. M. Zorzenon dos Santos, Physica A 231, 504 (1996); S. Moss de Oliveira, P.M.C. de Oliveira, and D. Stauffer, Brazilian Journal of Physics 26, 626 (1996).

[15] J. S. Sá Martins, S. M. de Oliveira, and G. A. de Medeiros, Phys. Rev. E 64, 1 (2001) 